\title{
Expectativas de estudiantes universitarios frente a sus capacidades y competencias para participar en cursos abiertos y en línea
}

\section{Expectations of College Students from their Skills and Competences to Participate in Open Online Courses}

\author{
Rosabel Roig-Vila \\ Universidad de Alicante. España. \\ rosabel.roig@ua.es \\ Marcelo Rioseco Pais \\ Universidad Católica del Maule. Chile. \\ mrioseco@ucm.cl \\ Marta Belmar Mellado \\ Universidad Católica del Maule. Chile. \\ mbelmar@ucm.cl
}

\begin{abstract}
Resumen
La educación virtual ha instalado una manera diferente de comprender la enseñanza y el aprendizaje. Asimismo, ha venido a modificar los roles de profesores y estudiantes y las formas de acceder al conocimiento. En este contexto, el estudio tiene como objetivo analizar las expectativas de estudiantes universitarios en torno a sus propias capacidades y competencias para participar en cursos abiertos y en línea. Nos aproximamos a estas cuestiones a través de un diseño descriptivo y correlacional con la participación de 115 estudiantes chilenos y 75 españoles. Los análisis psicométricos evidencian una buena consistencia del instrumento confirmando las dimensiones teóricas del instrumento, además de la correlación entre algunos factores y las variables demográficas de los grupos estudiados.
\end{abstract}

Palabras clave

E-learning, expectativas, competencias, educación superior, formación virtual

\begin{abstract}
Virtual education has installed a different way of understanding teaching and learning. Moreover, it has modified the roles of teachers and students, and ways to access knowledge. In this context, the study aims to analyze the expectations of college students about their own abilities and skills to participate in open online courses. We approach these issues through a descriptive and correlational design with the participation of 115 Chilean and 75 Spanish students. Psychometric analysis shows a good consistency of the instrument confirming its theoretical dimensions, in addition to the correlation between certain factors and demographic variables of the groups that were studied.
\end{abstract}

\section{Keywords}

E-learning, Expectations, Skills, Higher Education, Virtual Training 


\section{Introducción}

La masificación de las Tecnologías de la Información y la Comunicaciones (TIC) y, específicamente de Internet, es un hecho indiscutible en la actualidad. Según la empresa de servicios online Pingdom, se estima que en el 2012 (Internet World Stats, 2014) había en el planeta cerca de 2.400 millones de usuarios de Internet; Asia concentraba el 44,8\%, Europa poseía el 21,5\%, América del Norte el 11,4\%, y América del Sur el $10,6 \%$ de internautas.

Las instituciones educativas no son ajenas a este fenómeno, más bien al contrario. En las últimas décadas se han visto en la necesidad de incorporar estos recursos en todos los planos de su acción como entidades formadoras (UNESCO, 2010). Hoy en día, las TIC han dejado de ser una promesa, para ocupar un lugar importante, tanto en la forma como en el fondo en que se lleva a cabo el proceso de enseñanza-aprendizaje (Cabero, 2000). En este contexto, en diferentes partes del mundo, se ha desarrollado y se ha promovido el aprendizaje basado en las nuevas redes de comunicación y, en especial, en Internet (Castaño-Muñoz, Duart y Teresa, 2015), a través de diversas modalidades, como e-learning, b-learning, m-learning y, recientemente, MOOC (Massive Open Online Course) (Zapata, 2015), aunque, este último, posee todavía un desarrollo incipiente por lo que se refiere a nuevas propuestas de personalización o de aprendizaje basado en las emociones de la enseñanza online (Cheng, 2014).

En el caso de España, según la Escuela Europea de Dirección de Empresa (EUDE), la formación online no acaba de consolidarse. La tasa de abandono en programas de máster y posgrados llega al 35\% y al $90 \%$ en los denominados MOOC. En Estados Unidos, la tasa de abandono alcanza un $85 \%$ en esta modalidad de cursos masivos (Vizoso, 2013). En el caso de Chile, un gran número de universidades cuenta con algún tipo de plataforma virtual (Moodle, Claroline, Dokeos, WebCT, etc.) para apoyar con herramientas online la impartición de los cursos presenciales.

Para que la enseñanza virtual funcione adecuadamente, tanto profesores como estudiantes deben ser capaces de introducirse de lleno en plataformas, aulas y bibliotecas virtuales, foros y chats y necesitan tener una preparación suficiente para responder a las nuevas exigencias tecnológicas. La formación virtual basada en plataformas requiere de un esfuerzo difícil de calcular y exige una dedicación de tiempo importante (Eleusis.net, 2004). Para evitar la deserción es fundamental que las instituciones que desarrollan programas de formación en línea sean capaces de mantener la motivación de los estudiantes en todo momento (JISC, 2008).

De acuerdo a una investigación desarrollada por Eduardo García Teske en el año 2007 en Uruguay, una de las principales causas del abandono de los alumnos que cumplen estudios bajo la modalidad de e-learning se relaciona con las expectativas generadas a través de la oferta de cursos en línea, las que, muchas veces, superan o se apartan de lo que los cursos virtuales están en condiciones de entregar o de lo que los participantes están en condiciones de realizar (Teske, 2007),

Considerando que la motivación del estudiante para permanecer y finalizar los cursos virtuales es fundamental para su continuidad en los programas de formación en línea, el

Expectativas de estudiantes universitarios frente a sus capacidades y competencias para participar en cursos abiertos y en línea. Rosabel Roig-Vila Marcelo Rioseco Pais y Marta Belmar Mellado 
presente trabajo desarrolla una herramienta para detectar cuáles son las expectativas que los estudiantes tienen hacia sus propias competencias y capacidades para participar en cursos virtuales. Para tal efecto, toma en consideración las competencias TIC definidas por la UNESCO para docentes (UNESCO, 2008), las competencias genéricas establecidas por el Espacio Europeo de Educación Superior (EEES) y, en este mismo marco, la propuesta de contenidos propuesta por el proyecto ALFIN en relación a las principales competencias genéricas del EEES vinculadas a la alfabetización de la información.

\section{Competencias TIC en el contexto actual}

Como fundamentación teórica de esta investigación se han considerado, principalmente, las competencias TIC definidas por la UNESCO para docentes, las competencias genéricas establecidas por el Espacio Europeo de Educación Superior (EEES) y, en este mismo marco, la propuesta de contenidos propuesta por el proyecto ALFIN en relación a las principales competencias genéricas del EEES vinculadas a la alfabetización de la información. Cabe decir que la propuesta de la UNESCO se ha elegido ya que, a pesar de que está referida a docentes, el estudio se realiza con estudiantes universitarios de facultades de educación que serán, una vez egresados, docentes, y, por tanto, deben adquirir las competencias necesarias para tal dedicación profesional. Por último, se tomaron también muy en cuenta los resultados de un estudio cualitativo llevado a cabo por los investigadores Gabriel Valerio Ureña y Jaime Ricardo Valenzuela (Ureña y Valenzuela, 2011), del Instituto Tecnológico de Monterrey en torno a las competencias para el e-learning 2.0.

\subsection{Competencias TIC definidas por la UNESCO}

Basándose en el marco del plan de estudios elaborado por la UNESCO para el proyecto de los Estándares de Competencia en TIC para Docentes (UNESCO, 2008) para la elaboración del presente cuestionario, se consideró el enfoque relacionado con la generación de conocimiento en el componente de utilización de las TIC.

Expectativas de estudiantes universitarios frente a sus capacidades y competencias para participar en cursos abiertos y en línea. Rosabel Roig-Vila Marcelo Rioseco Pais y Marta Belmar Mellado 


\section{Figura 1}

\section{Competencias TIC UNESCO}

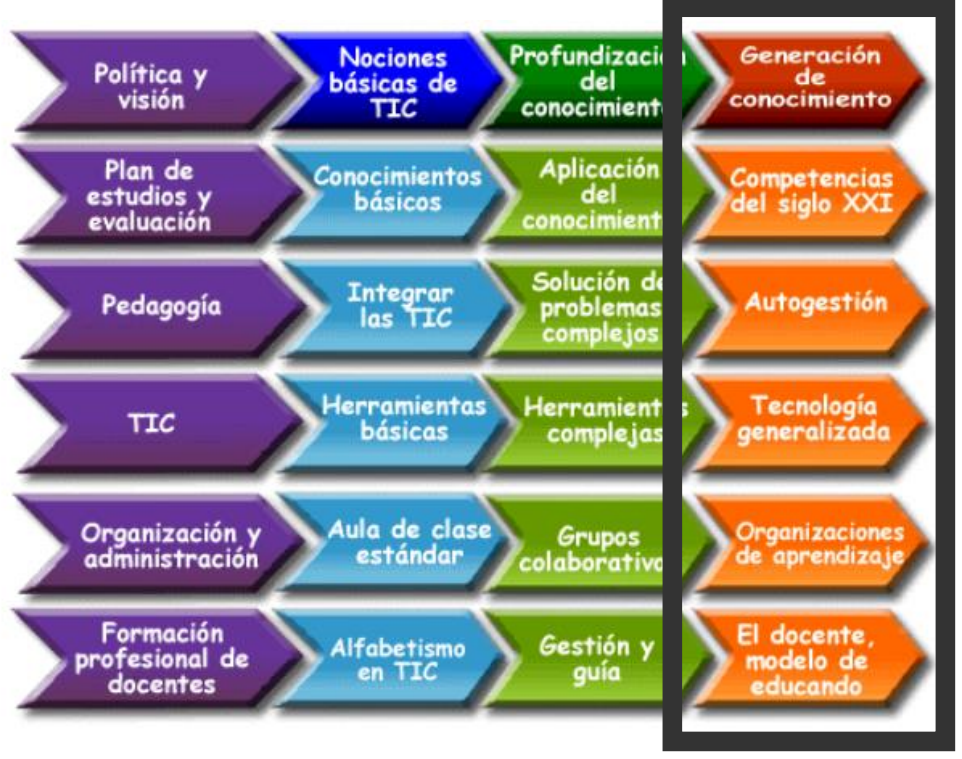

Los objetivos recomendados para incorporar en el plan de estudios (currículum) establecen el uso de tecnología generalizada, lo que significa, "producir conocimientos y aprender colaborativa y continuamente, [para lo cual] se utilizan múltiples dispositivos en red, además de recursos y contextos digitales" (p.17). Así mismo, las competencias docentes consideradas por la UNESCO sobre la base de este enfoque, determinan la "capacidad de diseñar comunidades de conocimiento basadas en las TIC, y también de saber utilizar estas tecnologías para apoyar el desarrollo de las habilidades de los estudiantes tanto en materia de creación de conocimientos como para su aprendizaje permanente y reflexivo" (p. 17).

\subsection{Competencias en el Espacio Europeo de Educación Superior (EEES)}

La creación del Espacio Europeo de Educación Superior responde al desarrollo de un proceso de convergencia en ámbitos como el monetario, económico, social, jurídico, educativo, cultural, etc. El EEES se constituye como una forma de concretar las necesarias reformas educativas. Para tal efecto, aparece la necesidad de adecuar la formación universitaria a las exigencias del mundo laboral (Fuentes, 2008). En este contexto, la reorganización del currículo debe centrarse y desarrollarse a partir de perfiles profesionales que sean capaces de conjugar la enseñanza científica con el desarrollo de competencias transversales.

En el marco del EEES, el Proyecto Tuning se ha convertido en un referente fundamental para la clasificación de competencias. Tuning diferencia entre competencias genéricas y específicas. Las primeras, identifican los elementos compartidos que pueden ser comunes a cualquier titulación (capacidad de aprender, de diseñar proyectos, destrezas administrativas); las segundas, deben estar relacionadas con cada área temática, con la especificidad propia de un campo de estudio o disciplina.

Ahora bien, las competencias genéricas pueden organizarse en tres criterios 0 modalidades:

Expectativas de estudiantes universitarios frente a sus capacidades y competencias para participar en cursos abiertos y en línea. Rosabel Roig-Vila Marcelo Rioseco Pais y Marta Belmar Mellado 
a) Competencias instrumentales. Se identifican con capacidades de carácter cognitivo, metodológico, tecnológico y lingüístico que posibilitan un desenvolvimiento académico básico al estudiante universitario. Son competencias que tienen una función instrumental y entre ellas podemos señalar:

a.1 Cognitivas: capacidad de comprender y utilizar ideas y pensamientos.

a.2 Metodológicas: capacidad para organizar el tiempo y las estrategias para el aprendizaje, tomar decisiones o resolver problemas.

a.3 Lingüísticas: tales como la comunicación oral y escrita o conocimiento de una segunda lengua

a.4 Tecnológicas: relacionadas con el uso del ordenador.

b) Competencias interpersonales. Son aquellas relacionadas con la capacidad de utilizar las habilidades comunicativas y críticas; en definitiva, aquellas capacidades que hacen que las personas logren una buena interacción con los demás. Se subdividen en: Individuales relativas a la capacidad de expresar los sentimientos, habilidades críticas y de autocrítica; y Sociales relacionadas con la capacidad de trabajar en equipo, o la expresión de compromiso social o ético. Estas competencias tienden a facilitar los procesos de interacción social y cooperación.

c) Competencias Sistémicas. Permiten aproximarse a la realidad en su complejidad de relaciones y no como un conjunto de hechos aislados. Conciernen a los sistemas como totalidad. Suponen una combinación de la comprensión, la sensibilidad y el conocimiento que permiten a la persona ver cómo las partes de un todo se relacionan y se agrupan. Entre ellas se incluyen la habilidad de planificar los cambios de manera que se puedan hacer mejoras en los sistemas. Es importante destacar que las competencias sistémicas o integradoras requieren como base la adquisición previa de competencias instrumentales e interpersonales.

\subsection{Proyecto ALFIN}

El proyecto ALFIN --habilidades y competencias de gestión de la información para aprender a aprender en el marco del espacio europeo de enseñanza superior-, es un portal educativo cuyo propósito es facilitar el entrenamiento y adquisición de habilidades, competencias y destrezas genéricas, relacionadas con todos los aspectos importantes de la gestión, acceso y uso de la información. Se basa en la experiencia acumulada en la creación del portal electrónico e-COMS (2004) y en la filosofía de los modelos Big Six (1995), Declaración de la Sorbona (1998), INTO Info (1998), Declaración de Bolonia (1999), PLUS (1999), CERICE (2000), ALA (2000), Declaración de Praga (2001), DESECO (2001), Unión Europea (2003), Declaración de Berlín (2003), iniciativa e-EUROPE (2003-05).

ALFIN ha seleccionado competencias genéricas instrumentales, interpersonales $\mathrm{y}$ sistémicas, estableciendo seis bloques de contenidos transversales:

- Aprende a aprender

Expectativas de estudiantes universitarios frente a sus capacidades y competencias para participar en cursos abiertos y en línea. Rosabel Roig-Vila Marcelo Rioseco Pais y Marta Belmar Mellado 
- Aprender a buscar y evaluar información

- Aprender a analizar y sintetizar

- Aprender a generar conocimiento

- Aprender a trabajar juntos

- Usar la tecnología para aprender

Para cumplir con su objetivo, el portal se vale de los siguientes recursos disponibles en línea y accesibles para todos los usuarios, de manera pública:

- Hipervínculos

- Esquemas

- Ejemplos interactivos con solución de problemas

- Material multimedia, para el aprendizaje y la autoevaluación

- Preguntas frecuentes, que aportarán soluciones a las cuestiones más problemáticas

- Correo electrónico, que facilitará las tutorías permanentes y personalizadas

- Foros, que proporcionarán un espacio para debatir virtualmente aspectos puntuales y contribuir a compartir el conocimiento.

\subsection{Competencias informáticas para el e-learning $\mathbf{2 . 0}$}

El concepto de e-learning 2.0 alude a una nueva generación del e-learning al incorporar software social o Web 2.0 en la educación electrónica. Entre otras fuentes, se sustenta en el conectivismo de Siemens (2004), un enfoque que, sin ser considerado todavía como una nueva teoría del aprendizaje, sí se apunta como tal a modo de propuesta, y que asegura que el e-learning tiene el potencial de ser personal, social y flexible, y ser una buena herramienta para apoyar la creación de comunidades de aprendizaje personalizadas, tales como blogs, wikis y otros programas sociales (Ureña y Valenzuela, 2011).

Considerando que la Web 2.0 hace referencia a una segunda generación de servicios de Internet, construida sobre la base de actividades de comunidades en línea que producen y comparten contenidos, el e-learning 2.0 es una segunda generación de espacios de enseñanza-aprendizaje en línea, basada en herramientas de software social, donde se potencia la colaboración, la comunicación en red y la interacción (Marcelo y Perera, 2007).

Desde esta perspectiva, la educación basada en el e-learning 2.0 podría "transformar el estilo de aprendizaje de las nuevas generaciones en la era de la información" (Ureña y Valenzuela, 2011): tradicionalmente se piensa que los estudiantes adquieren aprendizajes a través del estudio de libros y mediante su participación presencial en el aula. A través del e-learning 2.0, se superan las barreras de tiempo y espacio y se hace necesario explorar nuevos métodos de enseñanza.

Algunas de las principales diferencias entre el e-learning y el $e$-learning 2.0, se sintetizan en los siguientes puntos:

Expectativas de estudiantes universitarios frente a sus capacidades y competencias para participar en cursos abiertos y en línea. Rosabel Roig-Vila Marcelo Rioseco Pais y Marta Belmar Mellado 
- El estudiante crea y modifica los contenidos por medio de las herramientas que pone a su disposición la Web 2.0.

- El estudiante aprende gracias a la reutilización de contenidos que provienen de diversas fuentes y a conversaciones e interacciones con otros participantes.

- El aprendizaje proviene de diversos medios, tiende a ser informal y la formación es continua.

Ahora bien, está claro que una educación de este tipo requiere no sólo profesores preparados de otra manera (García-Valcárcel, Hernández y Recaman, 2012), sino estudiantes con habilidades, destrezas, conocimientos y actitudes diferentes a las de un alumno formado en la escuela tradicional (De Pablos, 2006). Las competencias necesarias para interactuar en ambientes de e-learning 2.0, además de las habilidades que permiten manejar información de manera efectiva y aquellas que permiten utilizar adecuadamente las TIC, requieren de una gran variedad de competencias cognitivas, motoras, sociales y emocionales.

En este contexto, los investigadores Gabriel Valerio Ureña y Jaime Ricardo Valenzuela (Ureña y Valenzuela, 2011), del Instituto Tecnológico de Monterrey, en México, llevaron a cabo un estudio de carácter naturalista para comprender y clasificar aquellas competencias informáticas necesarias para el e-learning 2.0. Este estudio utilizó el grupo de enfoque y la entrevista como herramientas de recolección de datos y el método de análisis de Lincoln y Guba (1985) para procesar los datos recogidos. Participaron en el estudio profesores con experiencia en el uso del e-learning y estudiantes egresados de la carrera de Licenciatura en Administración de Tecnologías de la Información, de Instituto Tecnológico de Monterrey.

Expectativas de estudiantes universitarios frente a sus capacidades y competencias para participar en cursos abiertos y en línea. Rosabel Roig-Vila Marcelo Rioseco Pais y Marta Belmar Mellado 


\section{Tabla 1}

Síntesis de competencias genéricas para el e-learning 2.0

\section{COMPETENCIAS GENÉRICAS INSTRUMENTALES}

EEES

Instituto tecnológico

- Capacidad de análisis y síntesis

- Capacidad de organización y planificación

- Comunicación oral y escrito en el idioma propio

- Resolución de problemas

- Habilidades de gestión de la información (capacidad para recuperar y analizar información de diversas fuentes)
E-learning 2.0 de Monterrey

Alfin EEES

- Enfocarse en una tarea

- Acceder a información de calidad

- Evaluar la calidad de la información

- Crear a partir de información existente

- Comunicar sus ideas en distintas formas analizar

- Leer para aprender Sintetizar Resumir
- Habilidades básicas informáticas

- Conocimientos de informática relativos al ámbito de estudio sintetizar y comunicar

- Aprender a

- Aprender a Segmentar

- Aprender a

- Aprender a Esquematizar

- Aprender a

- Aprender a Comunicar

- Aprender a Escribir

- Aprender a Citar

- Aprender a Presentar

Aprender a buscar $y$ evaluar información

- Aprender a buscar información

- Aprender a evaluar información

- Usar computación básica

- Manejar multimedios

- Usar tecnología Web

- Usar Web 2.0

Usar la tecnología para aprender

- Sistemas operativos

- Aplicaciones ofimáticas

- Herramientas de comunicación

- Entornos de
Expectativas en torno a propias capacidades $y$ competencias

- Comprensión de lectura

- Segmentar un texto en unidades con significado

- Sintetizar información

- Esquematizar información

- Resumir información

- Escribir y redactar

- Acceder a información de calidad

- Evaluar la calidad de la información

- Crear a partir de información existente

- Comunicar sus ideas en distintas formas

- Citar aprendizaje

- aplicaciones Web 2.0
- Sistemas operativos (Windows, Linux, Macintoch)

- Aplicaciones ofimáticas (Procesador de texto, hoja de cálculo, programa de presentaciones

- Herramientas de comunicación (navegadores y la world wide web (www), correo

Expectativas de estudiantes universitarios frente a sus capacidades y competencias para participar en cursos abiertos y en línea. Rosabel Roig-Vila Marcelo Rioseco Pais y Marta Belmar Mellado 
electrónico ,

listas de correo, foros, chats, vidoconferencia

S

- Entornos de aprendizaje (Moodle, Webct, Learning Space, Firts Class, etc.)

- Aplicaciones Web 2.0 (blogs, wikis, redes sociales, marcadores sociales, sitios para compartir recursos, guías sociales,

sindicación o

RSS, podcasting)

Tabla 2

Síntesis de competencias genéricas para el e-learning 2.0

INTERPERSONALES

EEES

- Trabajo en equipo

- Trabajo en un equipo de carácter interdisciplinar

- Capacidad para trabajar en un contexto internacional

- Capacidad para comunicarse con expertos de otros campos

- Habilidades en las relaciones interpersonales

- Compromiso ético

- Capacidad de crítica y autocritica

- $\quad$ Apreciación de la diversidad y multiculturalidad

\section{Alfin EEES} tecnológico de Monterrey

- Compartir conocimiento

- Respetar los derechos de autoría

- Discutir (intercambiar puntos de vista) sin agredir o sentirse agredido

$\begin{array}{cl}\text { Aprender a trabajar } \\ \text { juntos } & \text { Ética } \\ \bullet & \text { Aprender a } \\ & \text { trabajar en } \\ & \text { equipo } \\ \text { - } & \text { Toma de } \\ & \text { decisiones y } \\ & \text { negociación }\end{array}$

\section{Expectativas en torno a} propias capacidades $\mathbf{y}$ competencias

- Capacidad para trabajar en equipo

- Capacidad para comunicarse con expertos de otros campos

- Habilidades en las relaciones interpersonales

- Búsqueda de objetivos comunes en el curso y en los grupos de trabajo

- Respeto y apreciación de la diversidad y multiculturalidad

- Capacidad de crítica y autocritica

- Disposición a compartir información y conocimiento con otras personas

- Respeto a la autoría de la información y el conocimiento

Expectativas de estudiantes universitarios frente a sus capacidades y competencias para participar en cursos abiertos y en línea. Rosabel Roig-Vila Marcelo Rioseco Pais y Marta Belmar Mellado 
generado por otras personas

\section{Tabla 3}

Síntesis de competencias genéricas para el e-learning 2.0

\section{SISTÉMICAS}

\section{EEES}

- Capacidad para aplicar el conocimiento en la práctica

- Capacidad de aprendizaje (adquirir experiencia)

- Aprendizaje autónomo

- Adaptación a nuevas situaciones

- Liderazgo

- Conocimiento de otras culturas y costumbres

- Iniciativa y espíritu emprendedor

- Habilidades de investigación

- Capacidad para generar nuevas ideas (creatividad)

- Diseño y gestión de proyectos

\section{E-learning 2.0 Instituto tecnológico de Monterrey}

- Compromiso con el aprendizaje

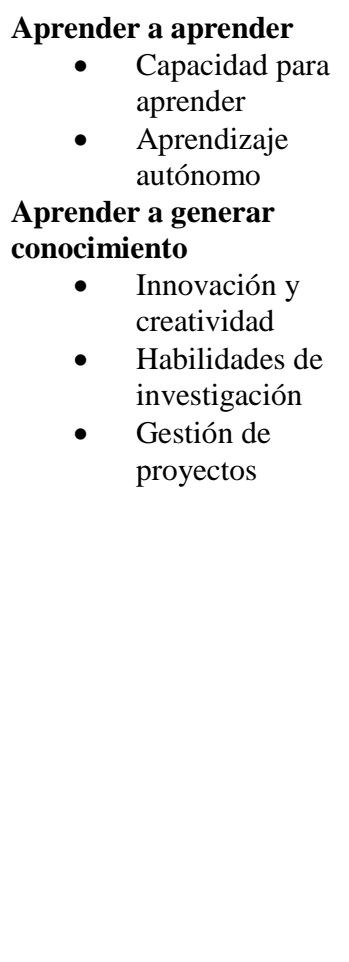

Alfin EEES

(1)


Universidad de Alicante, institución pública ubicada en la Comunidad Valenciana organizada en 7 Facultades con 21 licenciaturas, cuya matrícula es de más de 26.000 estudiantes. En el caso de Chile, participaron estudiantes de la Universidad Católica del Maule, ubicada en la región del Maule (zona centro sur de Chile). Esta institución pertenece al Consejo de Rectores de Chile y recibe aportes públicos. Actualmente tiene una matrícula de 6.200 estudiantes de pregrado adscritos a las titulaciones impartidas por las 8 Facultades que componen el marco educativo estructural.

En relación con los participantes de la universidad española, son estudiantes de un curso que les habilita lingüísticamente en la región para impartir docencia en educación secundaria. Se trata de una comunidad bilingüe donde un alto porcentaje de los establecimientos educativos siguen la línea de valenciano, de allí que requieran el curso "TIC i dinàmica d'aula aplicades al tractament integrat de llengües i continguts"1. En el caso de la universidad chilena, los estudiantes son de las titulaciones de Pedagogía en Educación Parvularia y Pedagogía en Educación Básica. Suelen denominarse a estas titulaciones "carreras de educación inicial” ya que los profesionales que egresan de estas carreras están habilitados para impartir docencia a niños en edad preescolar y educación primaria respectivamente.

\subsection{Tipo de muestreo y descripción de los participantes}

Para este estudio se optó específicamente por un muestreo no probabilístico de tipo intencional y sujeto a disponibilidad. La literatura señala que este tipo de muestreo se caracteriza porque la elección de las personas no depende de la probabilidad, sino de causas relacionadas con las características que otorga el propio investigador. En este sentido, la muestra depende del proceso de toma de decisiones del investigador asumiendo el sesgo que esta determinación pudiera tener (Hernández, Fernández y Baptista, 2010) y resulta apropiado cuando requiere no tanto una representatividad de elementos de una población, sino una cuidadosa y controlada elección de sujetos con ciertas características especificadas previamente, como es nuestro caso.

Participaron 115 estudiantes chilenos y 75 españoles. Cuando se les consultó sobre la formación previa, en el caso de la UCM 77 (66\%) cursaron la línea científico humanista y $38(33 \%)$ la línea técnico profesional; mientras que $25(33 \%)$ cursaron bachiller y 50 $(67 \%)$ una titulación universitaria. Otras variables ilustrativas de la muestra se presentan en la Tabla 1.

\footnotetext{
${ }^{1}$ El curso se denomina en castellano "TIC y dinámica del aula aplicadas al tratamiento integrado de lenguas y contenidos"
}

Expectativas de estudiantes universitarios frente a sus capacidades y competencias para participar en cursos abiertos y en línea. Rosabel Roig-Vila Marcelo Rioseco Pais y Marta Belmar Mellado 


\section{Tabla 4}

Variables demográficas

\begin{tabular}{lcccc}
\hline & \multicolumn{2}{c}{ UA } & \multicolumn{2}{c}{ UCM } \\
\hline Edad & Frecuencia & Porcentaje & Frecuencia & Porcentaje \\
Menor de 19 & 0 & 0 & 56 & 48 \\
Entre 20 y 24 & 28 & 37 & 51 & 44 \\
Entre 25 y 29 & 29 & 38 & 7 & 6 \\
Más de 30 & 18 & 24 & 1 & 1 \\
Titulación & & & 43 & 37 \\
Pedagogía E. Básica & - & - & 40 & 34 \\
(Talca) & - & - & 32 & 27 \\
E. Básica con mención & - & - & - & - \\
Educación Parvularia & 33 & 13 & - & - \\
Grado & 10 & 42 & - & - \\
Máster universitario & 32 & & & \\
Ninguno & Universidad Católica del Maule &
\end{tabular}

\section{Variables e instrumento}

Una variable es una característica, susceptible de observarse, que varía de acuerdo a los sujetos, pudiendo adoptar distintos valores. En el caso de este trabajo, desde el punto de vista conceptual, las expectativas que el estudiante posee hacia sus propias capacidades y competencias para participar de cursos abiertos y en línea, han sido abordadas a través de la definición de competencias genéricas instrumentales, interpersonales y sistémicas, delimitadas en los puntos anteriores.

Desde el punto de vista operativo, definiremos las siguientes cuatro variables:

3.1 Expectativa hacia competencias instrumentales TIC, mediante la puntuación obtenida a través del promedio de cinco ítems, para los cuales se utiliza una matriz de cinco valores (donde 5 representa el mayor grado y 1 el menor grado) sobre lo que el encuestado indica en relación al grado de preparación que considera que posee en torno a las expresiones contenidas en el cuestionario. Una puntuación alta en la escala indicaría que el estudiante posee un alto grado de expectativa hacia sus competencias instrumentales TIC. Por el contrario, una puntuación baja evidenciaría que tiene bajas expectativas en torno a sus competencias instrumentales TIC.

3.2 Expectativa hacia competencias instrumentales generales, mediante la puntuación obtenida a través del promedio de once ítems, para los cuales se utiliza una matriz de cinco valores (donde 5 representa el mayor grado y 1 el menor grado) sobre lo que el encuestado indica en relación al grado de capacidad que considera que posee en torno a las expresiones contenidas en el cuestionario. Una puntuación alta en la escala indicaría que el estudiante posee un alto grado de expectativa hacia sus competencias instrumentales generales. Por el contrario, una puntuación baja evidenciaría que tiene bajas expectativas en torno a sus competencias instrumentales generales.

3.3 Expectativa hacia competencias interpersonales, mediante la puntuación obtenida a través del promedio de ocho ítems, para los cuales se utiliza una matriz de cinco valores (donde 5 representa el mayor grado y 1 el menor grado) sobre lo que el

Expectativas de estudiantes universitarios frente a sus capacidades y competencias para participar en cursos abiertos y en línea. Rosabel Roig-Vila Marcelo Rioseco Pais y Marta Belmar Mellado 
encuestado indica en relación al grado de preparación y disposición que considera que posee en torno a las expresiones contenidas en el cuestionario. Una puntuación alta en la escala indicaría que el estudiante posee un alto grado de expectativa hacia sus competencias interpersonales. Por el contrario, una puntuación baja evidenciaría que tiene bajas expectativas en torno a sus competencias interpersonales.

3.4 Expectativa hacia competencias sistémicas, mediante la puntuación obtenida a través del promedio de doce ítems, para los cuales se utiliza una matriz de cinco valores (donde 5 representa el mayor grado y 1 el menor grado) sobre lo que el encuestado indica en relación al grado de competencia que considera que posee en torno a las expresiones contenidas en el cuestionario. Una puntuación alta en la escala indicaría que el estudiante posee un alto grado de expectativa hacia sus competencias sistémicas. Por el contrario, una puntuación baja evidenciaría que tiene bajas expectativas en torno a sus competencias sistémicas.

En síntesis, el cuestionario estuvo compuesto por un total de 36 ítems donde los estudiantes debían responder su grado de preparación; en este sentido, el valor 5 implicaba un mayor grado de preparación y 1 el menor grado. Para conocer tanto la fiabilidad como la consistencia interna del cuestionario, se calculó el estadístico Alpha de Cronbach, obteniéndose un índice de 0.94 para el total de la muestra. Según Hernández, Fernández y Baptista (2010), la puntuación superior a 0.9 significa una confiabilidad elevada, por lo que el instrumento cumple con el propósito de medir coherentemente lo que se marcó como objetivo. Para examinar la validez de constructo se llevó a cabo un análisis factorial exploratorio utilizando como método de extracción el análisis de componentes principales (rotación varimax). El índice de adecuación muestral $(\mathrm{KMO}=, 90)$, así como la prueba de esfericidad de Bartlett, [ $\chi_{2}(630)$ $=3906,15 ; p<, 000]$, resultaron muy adecuados, dando apoyo a la factorización de la matriz de correlaciones. El análisis de componentes principales reveló la presencia de cuatro factores que explican el 54,49\% de la varianza: competencias instrumentales en uso TIC (F4) $(10,76 \%)$ con 5 ítems, competencias instrumentales generales (F1) $(17,08 \%)$ con 11 ítems, competencias interpersonales $(\mathrm{F} 3)(11,65 \%)$ con 8 ítems y competencias sistémicas $(14,9 \%)$ con 12 ítems.

En el análisis de fiabilidad de los elementos que componen el factor "Expectativas hacia competencias instrumentales TIC" (véase tabla 2), se observa que los índices de Alfa de Cronbach, si el ítem se elimina, fluctúan entre ,776 y ,820, es decir se presentan por debajo del índice de consistencia interna del factor, que corresponde a ,820. Se confirma así que los elementos aportan fiabilidad al instrumento.

Expectativas de estudiantes universitarios frente a sus capacidades y competencias para participar en cursos abiertos y en línea. Rosabel Roig-Vila Marcelo Rioseco Pais y Marta Belmar Mellado 
Tabla 5

Análisis de fiabilidad para el factor "Expectativas hacia competencias instrumentales TIC"

\begin{tabular}{|c|c|c|c|}
\hline Elementos & M & $\mathbf{V}$ & $\square$ \\
\hline Sistemas operativos (Windows, Linux, Macintoch) & 11,57 & 11,463 & ,776 \\
\hline $\begin{array}{l}\text { Aplicaciones ofimáticas (Procesador de texto, hoja de cálculo, programa de } \\
\text { presentaciones }\end{array}$ & 11,57 & 11,929 & ,789 \\
\hline $\begin{array}{l}\text { Herramientas de comunicación (navegadores y la world wide web (www), } \\
\text { correo electrónico, listas de correo, foros, chats, videoconferencias) }\end{array}$ & 10,59 & 13,905 &, 820 \\
\hline Entornos de aprendizaje (Moodle, Webct, Learning Space, Firts Class, etc) & 12,66 & 12,459 & ,792 \\
\hline $\begin{array}{l}\text { Aplicaciones Web } 2.0 \text { (blogs, wikis, redes sociales, marcadores sociales, } \\
\text { sitios para compartir recursos, guías sociales, sindicación o RSS, podcasting) }\end{array}$ & 12,20 & 11,632 & ,781 \\
\hline
\end{tabular}

En el análisis de fiabilidad de los elementos que componen el factor "Expectativas hacia competencias instrumentales generales" (véase tabla 3), se observa en los índices de Alfa de Cronbach que si el ítem se elimina, fluctúan entre ,902 y ,917, es decir, se presentan igual o por debajo del índice de consistencia interna del factor, que corresponde a ,914. La única excepción la constituye la capacidad de citar, que se sitúa levemente por encima de este valor. Se confirma así que los elementos aportan fiabilidad al instrumento.

Tabla 6

Análisis de fiabilidad para el factor "Expectativas hacia competencias instrumentales generales"

\begin{tabular}{lccc}
\hline \multicolumn{1}{c}{ Elemento } & M & V & $\square$ \\
Comprensión de lectura & 35,88 & 45,938 &, 906 \\
Segmentar un texto en unidades con significado & 36,37 & 44,743 &, 903 \\
Sintetizar información & 36,13 & 45,487 &, 904 \\
Esquematizar información & 36,27 & 44,105 &, 902 \\
Resumir información & 35,99 & 45,455 &, 903 \\
Escribir y redactar & 35,95 & 46,611 &, 910 \\
Acceder a información de calidad & 36,15 & 47,015 &, 910 \\
Evaluar la calidad de la información & 36,22 & 45,856 &, 907 \\
Crear a partir de información existente & 36,14 & 45,632 &, 903 \\
Comunicar sus ideas en distintas formas & 36,09 & 45,479 &, 906 \\
Citar & 36,60 & 46,686 &, 917 \\
\hline
\end{tabular}

En el análisis de fiabilidad de los elementos que componen el factor "Expectativas hacia competencias interpersonales" (véase tabla 4), se observa que los índices de Alfa de Cronbach, si el ítem se elimina, fluctúan entre ,821 y ,849, es decir se presentan por debajo del índice de consistencia interna del factor, que corresponde a ,858. Se confirma así que los elementos aportan fiabilidad al instrumento.

Expectativas de estudiantes universitarios frente a sus capacidades y competencias para participar en cursos abiertos y en línea. Rosabel Roig-Vila Marcelo Rioseco Pais y Marta Belmar Mellado 
Tabla 7

Análisis de fiabilidad para el factor "Expectativas hacia competencias interpersonales"

\begin{tabular}{lccc}
\hline \multicolumn{1}{c}{ Elemento } & $\mathbf{M}$ & $\mathbf{V}$ & $\square$ \\
Capacidad para trabajar en equipo & 28,42 & 16,371 &, 840 \\
Capacidad para comunicarse con expertos de otros campos & 29,22 & 16,459 &, 847 \\
Habilidades en las relaciones interpersonales & 28,90 & 16,567 &, 849 \\
Búsqueda de objetivos comunes en el curso y en los grupos de trabajo & 28,89 & 15,030 &, 821 \\
Respeto y apreciación de la diversidad y multiculturalidad & 28,18 & 17,259 &, 847 \\
Capacidad de crítica y auto-critica & 28,61 & 16,620 &, 844 \\
Disposición a compartir información y conocimiento con otras personas & 28,39 & 16,473 &, 839 \\
$\begin{array}{l}\text { Respeto a la autoría de la información y el conocimiento generado por otras } \\
\text { personas }\end{array}$ & 28,36 & 16,656 &, 839 \\
\hline
\end{tabular}

En el análisis de fiabilidad de los elementos que componen el factor "Expectativas hacia competencias sistémicas" (véase tabla 5), se observa en los índices de Alfa de Cronbach que si el ítem se elimina, fluctúan entre ,870 y ,878, es decir, se presentan por debajo del índice de consistencia interna del factor, que corresponde a ,880. Se confirma así que los elementos aportan fiabilidad al instrumento.

Tabla 8

Análisis de fiabilidad para el factor "Expectativas hacia competencias sistémicas"

\begin{tabular}{lccc}
\hline \multicolumn{1}{c}{ Elemento } & $\mathbf{M}$ & $\mathbf{V}$ & $\square$ \\
Compromiso con mi propio aprendizaje & 42,69 & 38,763 &, 873 \\
Disposición y capacidad para enfocarme y concentrarme en una tarea & 43,07 & 37,423 &, 872 \\
Capacidad para aprender & 42,89 & 37,496 &, 866 \\
Capacidad de aprendizaje autónomo & 43,11 & 37,322 &, 868 \\
Capacidad para aplicar el conocimiento en la práctica & 43,02 & 37,227 &, 866 \\
Adaptación a nuevas situaciones & 43,05 & 37,802 &, 872 \\
Liderazgo & 43,59 & 36,634 &, 878 \\
Conocimiento de otras culturas y costumbres & 43,48 & 37,902 &, 876 \\
Iniciativa y espíritu emprendedor & 43,14 & 36,493 &, 867 \\
Habilidades de investigación & 43,45 & 37,328 &, 871 \\
Capacidad para generar nuevas ideas (creatividad) & 43,21 & 37,045 &, 867 \\
Diseño y gestión de proyectos & 43,44 & 36,735 &, 870 \\
\hline
\end{tabular}

\section{Resultados}

Una vez determinada la fiabilidad de la escala de expectativas de los estudiantes universitarios, interesaba conocer en qué factores los estudiantes manifestaban mayor o menor preparación, y por otra parte, determinar si existía alguna relación entre las variables demográficas y los factores de la escala.

Expectativas de estudiantes universitarios frente a sus capacidades y competencias para participar en cursos abiertos y en línea. Rosabel Roig-Vila Marcelo Rioseco Pais y Marta Belmar Mellado 
Según podemos ver en la tabla 6 respecto al factor "competencias instrumentales", los alumnos se sienten mejor preparados con las herramientas de comunicación, y menos preparados con los entornos de aprendizaje.

Tabla 9

Estadísticos descriptivos "Competencias instrumentales TIC"

\begin{tabular}{lccccc}
\hline & $\mathrm{N}$ & Mín & Máx & Media & D. Estándar \\
Sistemas operativos & 190 & 1 & 5 & 3,07 & 1,197 \\
Aplicaciones ofimáticas & 190 & 1 & 5 & 3,08 & 1,163 \\
Herramientas de & 190 & 1 & 5 & 4,06 &, 933 \\
$\begin{array}{l}\text { comunicación } \\
\text { Entornos de aprendizaje }\end{array}$ & 190 & 1 & 5 & 1,99 & 1,079 \\
Aplicaciones Web 2.0 & 190 & 1 & 5 & 2,45 & 1,184 \\
N válido (por lista) & 190 & & & & \\
\hline
\end{tabular}

En el factor "competencias instrumentales generales" (véase tabla 7) las medias indican que los estudiantes declaran mayor preparación en las competencias de comprensión lectora $(3,90)$, escritura y redacción $(3,83)$ y en resumir información $(3,78)$; mientras que su menor desarrollo estaría en la competencia que implica hacer citas, sin que esta se aleje considerablemente de la media general del factor $(3,18)$.

Tabla 10

Estadísticos descriptivos "Competencias instrumentales generales"

\begin{tabular}{|c|c|c|c|c|c|}
\hline & $\mathrm{N}$ & Mín & Máx & Media & D. estándar \\
\hline Comprensión de lectura & 190 & 1 & 5 & 3,90 & ,888 \\
\hline $\begin{array}{l}\text { Segmentar un texto en } \\
\text { unidades con significado }\end{array}$ & 190 & 1 & 5 & 3,41 & ,931 \\
\hline Sintetizar información & 190 & 1 & 5 & 3,65 & ,882 \\
\hline Esquematizar información & 190 & 1 & 5 & 3,51 & ,975 \\
\hline Resumir información & 190 & 2 & 5 & 3,78 &, 855 \\
\hline Escribir y redactar & 190 & 1 & 5 & 3,83 & ,910 \\
\hline $\begin{array}{l}\text { Acceder a información de } \\
\text { calidad }\end{array}$ & 190 & 1 & 5 & 3,63 &, 874 \\
\hline $\begin{array}{l}\text { Evaluar la calidad de la } \\
\text { información }\end{array}$ & 190 & 1 & 5 & 3,56 & ,923 \\
\hline $\begin{array}{l}\text { Crear a partir de } \\
\text { información existente }\end{array}$ & 190 & 2 & 5 & 3,64 &, 847 \\
\hline $\begin{array}{l}\text { Comunicar sus ideas en } \\
\text { distintas formas }\end{array}$ & 190 & 1 & 5 & 3,69 & ,933 \\
\hline Citar & 190 & 1 & 5 & 3,18 & 1,054 \\
\hline $\mathrm{N}$ válido (por lista) & 190 & & & & \\
\hline
\end{tabular}

Se observa en la tabla 8, que en el factor "competencias interpersonales" los estudiantes

Expectativas de estudiantes universitarios frente a sus capacidades y competencias para participar en cursos abiertos y en línea. Rosabel Roig-Vila Marcelo Rioseco Pais y Marta Belmar Mellado 
se sienten mejor preparados para respetar y apreciar la diversidad y multiculturalidad $(4,53)$ y menos preparados para poner en práctica sus habilidades en las relaciones interpersonales $(3,81)$.

\section{Tabla 11}

Estadísticos descriptivos "Competencias interpersonales"

\begin{tabular}{|c|c|c|c|c|c|}
\hline & $\mathrm{N}$ & Mín & Máx & Media & D. estándar \\
\hline $\begin{array}{l}\text { Capacidad para trabajar } \\
\text { en equipo }\end{array}$ & 190 & 2 & 5 & 4,29 & ,808 \\
\hline $\begin{array}{l}\text { Capacidad para } \\
\text { comunicarse con expertos } \\
\text { de otros campos }\end{array}$ & 190 & 1 & 5 & 3,49 &, 853 \\
\hline $\begin{array}{l}\text { Habilidades en las } \\
\text { relaciones interpersonales }\end{array}$ & 190 & 1 & 5 & 3,81 & ,858 \\
\hline $\begin{array}{l}\text { Búsqueda de objetivos } \\
\text { comunes en el curso y en } \\
\text { los grupos de trabajo }\end{array}$ & 190 & 1 & 5 & 3,82 & ,885 \\
\hline $\begin{array}{l}\text { Respeto y apreciación de } \\
\text { la diversidad y } \\
\text { multiculturalidad }\end{array}$ & 190 & 2 & 5 & 4,53 &, 718 \\
\hline $\begin{array}{l}\text { Capacidad crítica y } \\
\text { autocrítica }\end{array}$ & 190 & 2 & 5 & 4,10 & 807 \\
\hline $\begin{array}{l}\text { Disposición a compartir } \\
\text { información y } \\
\text { conocimiento con otras } \\
\text { personas }\end{array}$ & 190 & 2 & 5 & 4,32 & ,780 \\
\hline $\begin{array}{l}\text { Respeto a la autoría de la } \\
\text { información y el } \\
\text { conocimiento generado } \\
\text { por otras personas }\end{array}$ & 190 & 2 & 5 & 4,35 & ,753 \\
\hline $\mathrm{N}$ válido (por lista) & 190 & & & & \\
\hline
\end{tabular}

Igualmente, respecto al factor de "competencias sistémicas" (véase tabla 9), se observa mayor preferencia por la competencia de compromiso con su aprendizaje y con la capacidad de aprender, mientras que declaran menor preferencia con la competencia de liderazgo.

\section{Tabla 12}

Expectativas de estudiantes universitarios frente a sus capacidades y competencias para participar en cursos abiertos y en línea. Rosabel Roig-Vila Marcelo Rioseco Pais y Marta Belmar Mellado 
Estadísticos descriptivos "Competencias sistémicas"

\begin{tabular}{|c|c|c|c|c|c|}
\hline & $\mathrm{N}$ & Mín & Máx & Media & D. estándar \\
\hline $\begin{array}{l}\text { Compromiso con mi } \\
\text { propio aprendizaje }\end{array}$ & 190 & 1 & 5 & 4,41 & ,705 \\
\hline $\begin{array}{l}\text { Disposición y capacidad } \\
\text { para enfocarme y } \\
\text { concentrarme en una tarea }\end{array}$ & 190 & 1 & 5 & 4,03 & ,866 \\
\hline Capacidad para aprender & 190 & 3 & 5 & 4,22 &, 713 \\
\hline $\begin{array}{l}\text { Capacidad de aprendizaje } \\
\text { autónomo }\end{array}$ & 190 & 2 & 5 & 4,00 & ,797 \\
\hline $\begin{array}{l}\text { Capacidad para aplicar el } \\
\text { conocimiento en la } \\
\text { práctica }\end{array}$ & 190 & 2 & 5 & 4,08 &, 758 \\
\hline $\begin{array}{l}\text { Adaptación a nuevas } \\
\text { situaciones }\end{array}$ & 190 & 2 & 5 & 4,06 &, 811 \\
\hline Liderazgo & 190 & 1 & 5 & 3,51 & 1,043 \\
\hline $\begin{array}{l}\text { Conocimiento de otras } \\
\text { culturas y costumbres }\end{array}$ & 190 & 1 & 5 & 3,62 &, 887 \\
\hline $\begin{array}{l}\text { Iniciativa y espíritu } \\
\text { emprendedor }\end{array}$ & 190 & 1 & 5 & 3,96 &, 881 \\
\hline $\begin{array}{l}\text { Habilidades de } \\
\text { investigación }\end{array}$ & 190 & 1 & 5 & 3,65 &, 858 \\
\hline $\begin{array}{l}\text { Capacidad para generar } \\
\text { nuevas ideas }\end{array}$ & 190 & 1 & 5 & 3,89 &, 810 \\
\hline $\begin{array}{l}\text { Diseño y gestión de } \\
\text { proyectos }\end{array}$ & 190 & 1 & 5 & 3,66 & ,910 \\
\hline $\mathrm{N}$ válido (por lista) & 190 & & & & \\
\hline
\end{tabular}

Una vez determinados los ítems en que los estudiantes lograban mayor preferencia o bien que se sentían más competentes, interesaba determinar la presencia o ausencia de correlación entre las variables demográficas y los factores de la escala.

En tal sentido, se observa que existe correlación significativa entre la variable "sexo" y las "competencias instrumentales TIC" $(, 019)$, y con la "edad" $(, 005)$.

Del mismo modo se puede observar que existe correlación significativa entre la variable "competencias instrumentales TIC" y las "competencias instrumentales generales" $(, 000)$; “competencias interpersonales" $(, 000)$; “competencias sistémicas" $(, 000)$ y con la" edad" $(, 000)$.

También se aprecia que hay correlación entre la variable "competencias instrumentales generales" y las "competencias interpersonales" (,000); "competencias sistémicas" $(, 000)$ y con la "edad" (,003). Mientras que las "competencias interpersonales" solo correlacionan con las "competencias sistémicas" $(, 000)$.

Expectativas de estudiantes universitarios frente a sus capacidades y competencias para participar en cursos abiertos y en línea. Rosabel Roig-Vila Marcelo Rioseco Pais y Marta Belmar Mellado 
Tabla 13

Correlaciones entre variables demográficas y factores de la escala

\begin{tabular}{|c|c|c|c|c|c|c|}
\hline Sexo & Sexo & $\begin{array}{l}\text { Competencias } \\
\text { instrumentales } \\
\text { TIC } \\
-, 170^{*}\end{array}$ & $\begin{array}{l}\text { Competencias } \\
\text { instrumentales } \\
\text { generales } \\
-, 133\end{array}$ & $\begin{array}{l}\text { Competencias } \\
\text { interpersonales } \\
-, 017\end{array}$ & $\begin{array}{l}\text { Competencia } \\
\text { s sistémicas } \\
-, 028\end{array}$ &,$- 204 * *$ \\
\hline $\begin{array}{l}\text { Competencias } \\
\text { instrumentale } \\
\text { s TIC }\end{array}$ &,$- 170 *$ & 1 &, $571 * *$ &, $288 * *$ &, $434 * *$ &, $306 * *$ \\
\hline $\begin{array}{l}\text { Competencias } \\
\text { instrumentales } \\
\text { generales }\end{array}$ &,- 133 &, $571 * *$ & 1 &, $566 * *$ &, $624 * *$ &, $213 * *$ \\
\hline $\begin{array}{l}\text { Competencias } \\
\text { interpersonales }\end{array}$ &,- 017 & ,288** &, $566 * *$ & 1 &, $624 * *$ &, 050 \\
\hline $\begin{array}{l}\text { Competencias } \\
\text { sistémicas }\end{array}$ &,- 028 &, $434 * *$ &, $624 * *$ &, $624 * *$ & 1 &,- 030 \\
\hline Edad &,$- 204 * *$ &, $306 * *$ &, $213 * *$ &, 050 &,- 030 & 1 \\
\hline
\end{tabular}

*. La correlación es significativa en el nivel 0,005 (2 colas).

**. La correlación es significativa en el nivel 0,001 (2 colas).

\section{Conclusiones}

Teniendo en cuanta los análisis realizados en esta investigación, se puede concluir que, a partir de la escala de expectativas de estudiantes universitarios en torno a sus capacidades y competencias para participar en cursos abiertos y en línea, se han obtenido evidencias de validez sobre cuatro dimensiones de las competencias informáticas necesarias para el e-learning, todo ello a partir de la exploración de las propiedades psicométricas de la escala en una muestra de estudiantes chilenos y españoles.

A nivel general se han obtenido indicadores adecuados, es decir, las propiedades psicométricas de los ítems son adecuadas (Carrero-Dios y Pérez, 2005). Esto permite concluir que la escala de expectativas para estudiantes universitarios en torno a sus capacidades y competencias para participar en cursos abiertos y en línea presenta características psicométricas aceptables y que en general, muestra una adecuada consistencia interna.

A partir del estudio de la estructura interna del instrumento, es posible definir cuatro factores que explican las "competencias instrumentales TIC", las "competencias instrumentales generales", las "competencias interpersonales" y las "competencias sistémicas", tanto para alumnos españoles de pedagogía como para los estudiantes chilenos. Dichos factores explican el $54,4 \%$ del total de la varianza, con un coeficiente Alpha de ,94.

La correlación obtenida entre los factores de la escala y las variables demográficas confirman la utilidad del instrumento, dando cuenta de la asociación lineal entre algunas de las variables estudiadas, y que dicha asociación muestra una elevada correlación directa. De esta forma, el sexo de los estudiantes encuestados está en relación directa

Expectativas de estudiantes universitarios frente a sus capacidades y competencias para participar en cursos abiertos y en línea. Rosabel Roig-Vila Marcelo Rioseco Pais y Marta Belmar Mellado 
con las competencias instrumentales TIC (de ofimática, entornos web, sistemas operativos, etc.) y con la edad de los estudiantes, ya que la mayoría de los estudiantes se ubica en el rango de edad de 20 a 29 años, lo que implica que este grupo etario ha ido creciendo y desarrollando sus competencias en función de las exigencias pedagógicas, tecnológicas y sociales que el entorno les exige.

Por otra parte, las competencias instrumentales TIC se asocian con las competencias instrumentales generales (comprensión lectora, escritura, síntesis, acceso a la información, comunicar ideas, etc.), con las competencias interpersonales (habilidades para entablar relaciones interpersonales, capacidad para trabajar en equipo, disposición para compartir información, etc.), con las competencias sistémicas (condiciones para el aprendizaje, liderazgo, adaptación a nuevas situaciones, adaptación a otras culturas y contextos, habilidades para investigar, etc.) y con la edad. Ello supone que en la medida en que los estudiantes desarrollan habilidades personales necesarias para enfrentar entornos de aprendizaje, simultáneamente van adquiriendo o movilizan recursos que les permiten socializar, intercambiar opiniones y ejecutar acciones con grupos de pares, ya sea en entornos virtuales o reales (Loughran, 2002). Igualmente la disposición frente al aprendizaje, y las actitudes de liderazgo que los jóvenes asumen, se ve influida por las herramientas TIC que manejan y por su edad. Esta población ha crecido en entornos comunicacionales menos convencionales, por lo cual mostrarían mayores aperturas y dominio de ciertos códigos lingüísticos necesarios para entablar nexos en sus respectivos espacios.

Las competencias instrumentales generales muestran una asociación positiva con las competencias interpersonales, con las sistémicas y con la edad; ello da cuenta también de que algunas condiciones cognitivas (resumir, escribir, leer, etc) y metacognitivas (síntesis, esquematizar, segmetar, etc) comunes para el aprendizaje en general, son necesarias también para comunicarse con los pares, con la familia, con el ambiente educativo y laboral, y para construir, no solo entornos educativos, sino también para establecer nuevas interacciones adecuadas a las demandas que la sociedad exige. Por su parte las competencias interpersonales muestran una asociación positiva con las competencias sistémicas, puesto que ambas competencias promueven habilidades y capacidades que conllevan a mejorar los procesos formativos personales, adaptándose a nuevas situaciones, y a la legitimación de otras culturas y contextos formativos. Este último resultado se corresponde con el estudio llevado a cabo por Rubia, Jorri y Anguita (2009), donde se destaca la importancia de las relaciones sociales y la interacción con el otro en la adquisición de conocimiento. Saber trabajar en grupo para conseguir objetivos comunes aparece como una competencia transversal de aprendizaje en todos los niveles de enseñanza.

Se puede concluir que la escala se muestra estable en dos países con culturas diferentes, tratándose además de una escala fiable, con una dimensionalidad que replica el contenido teórico y unos indicadores de consistencia interna y validez de constructo adecuados, además de una validez consecuencial que intenta responder a las exigencias sociales y orientar a la toma de decisiones. Los efectos de la validez consecuencial movilizan hacia un cambio social de desarrollo y continuación de programas e-learning, con grandes implicaciones en las políticas sociales y educacionales, repercutiendo, además, en la dinámica y organización de los centros educativos, como también en la

Expectativas de estudiantes universitarios frente a sus capacidades y competencias para participar en cursos abiertos y en línea. Rosabel Roig-Vila Marcelo Rioseco Pais y Marta Belmar Mellado 
mejora de las relaciones interpersonales en el entorno educativo y social.

Si bien los análisis realizados permiten contar con un instrumento fiable para abordar el estudio de las competencias e-learning en el contexto universitario chileno y español, sería de gran relevancia estudiar el comportamiento de las variables en una población más amplia, similar o distinta a la del estudio y contrastar los resultados para validar el instrumento. Aunque los resultados alcanzados aportan información valiosa sobre las competencias e-learning de los estudiantes de pedagogía, merecen ser analizados detalladamente en una población más amplia. ¿Se mantiene la fiabilidad del instrumento al incrementar la muestra?, ¿difieren o se asemejan las concepciones de competencias entre estudiantes y egresados de maestría?, ¿es el contexto sociocultural un buen indicador de las competencias e-learning?, cuestiones, que entre otras, serían de interés para abordarlas en futuras investigaciones.

Presentación del artículo: 20 de septiembre de 2015

Fecha de aprobación: 28 de septiembre de 2015

Fecha de publicación: 30 de octubre de 2015

Roig-Vila, R., Rioseco-Pais, M. y Belmar-Mellado, M.(2015). Expectativas de estudiantes universitarios frente a sus capacidades y competencias para participar en cursos abiertos y en línea. RED. Revista de Educación a Distancia. Número, 47(2). 30 de Octubre de 2015. Consultado el (dd/mm/aa) en http://www.um.es/ead/red/47

\section{Bibliografía}

Cabero J., Salinas, J., Duarte, A. y Domingo, J. (2000). Nuevas Tecnologías Aplicadas a la Educación. Madrid: Ed. Síntesis.

Carretero-Dios, H. y Pérez, C. (2005). Normas para el desarrollo y revisisón de estudios instrumentales. International Journal of Clinical and Health Psychology, 5, 521-551.

Castaño-Muñoz, J., Duart, J., y Teresa, S. (2015). Determinants of Internet use for interactive learning: an exploratory study. Journal of New Approaches In Educational Research, 4(1), 24-31. doi:10.7821/naer.2015.1.93

Cheng, C.Y J. (2014). An Exploratory Study of Emotional Affordance of a Massive Open Online Course. European Journal of Open, Distance and e Learning, 17(1), 43-55. http://www.eurodl.org/materials/contrib/2014/Cheng.pdf

De Pablos, J. (2006). El marco del impacto de las Tecnologías de la Información: herramientas conceptuales para interpretar la mediación tecnológica. Telos: Cuadernos de Comunicación, tecnología y sociedad, 67, 68-74.

Eleusis.net (2004). El futuro del eLearning: análisis del mercado y del contexto actual del eLearning. Peñaranda de Bracamonte: Fundación Germán Sánchez Ruipérez. http://www.fundaciongsr.es/pdfs/elearning.pdf

Expectativas de estudiantes universitarios frente a sus capacidades y competencias para participar en cursos abiertos y en línea. Rosabel Roig-Vila Marcelo Rioseco Pais y Marta Belmar Mellado 
Fuentes, C. (2008). Modelo de Comunicación para la Enseñanza a Distancia en Internet . Análisis Experimental de una Plataforma de E-Learning (Disertación doctoral no publicada). Universidad Autónoma de Barcelona, España.

García-Valcárcel, A., Hernández, A. y Recaman, A. (2012). La metodología del aprendizaje colaborativo a través de las TIC: una aproximación a las opiniones de profesores y alumnos. Revista Complutense de Educación, 23(1), 161-188.

Hernández, R., Fernández, C. \& Baptista, M. (2010). Metodología de la Investigación, Quinta Edición. Editorial Mc. Graw-Hill, México.

Internet World Stats (2014). Información extraída el 2 de febrero de 2014 de http://www.internetworldstats.com/stats.htm

JISC (2008). Great Expectations of ICT. How Higher Education Institutions are measuring up. http://www.jisc.ac.uk/media/documents/publications/jiscgreatexpectationsfinalreportj une08.pdf

Lincoln, Y.S. \& Guba, E. G. (1985). Naturalistic Inquiry. Beberly Hills, CA: Sage.

Loughran, J. (2002). Effective Reflective Practice: In Search of Meaning in Learning about Teaching. Journal of Teacher Education, 53(1), 33-43. DOI: $10.1177 / 0022487102053001004$

Marcelo, C. y Perera, V. (2007). Comunicación y aprendizaje electrónico: la interacción didáctica en los nuevos espacios virtuales de aprendizaje. Revista de Educación, 343, 381-429. Universidad de Sevilla, España. http://www.revistaeducacion.mec.es/re343/re343_17.pdf

Moreno-Murcia, J., Silveira Torregrosa, Y., y Belando Pedreño, N. (2015). Questionnaire evaluating teaching competencies in the university environment. Evaluation of teaching competencies in the university. Journal of New Approaches In Educational Research, 4(1), 54-61. doi:10.7821/naer.2015.1.106

Rubia, B., Jorri, I. y Anguita, R. (2009). Aprendizaje colaborativo y TIC. En J. De Pablos (Coord.), Tecnología Educativa. La formación del profesorado en la era digital. Málaga: Aljibe.

Siemens, G. (2004). Conectivismo: una Teoría de Aprendizaje para la Era Digital.Información extraída el 2 de febrero de 2014 de https://docs.google.com/document/d/1ZkuAzdx1191DgcC1E_XSmPTOk6Gu1K2SEvXtduG3gc/edit

Teske, E. (2007). El "abandono" en cursos de e-learning: algunos aprendizajes para nuevas propuestas. Revista Iberoamericana de Educación, 44/3, 1-16.

UNESCO (2008). Estándares de Competencias TIC para Docentes. París: UNESCO.

Expectativas de estudiantes universitarios frente a sus capacidades y competencias para participar en cursos abiertos y en línea. Rosabel Roig-Vila Marcelo Rioseco Pais y Marta Belmar Mellado 


\section{http://www.oei.es/tic/UNESCOEstandaresDocentes.pdf}

UNESCO (2010). Informe mundial sobre el aprendizaje y la educación de adultos. Hamburgo: Instituto de la UNESCO para la Educación a lo Largo de Toda la Vida http://www.unesco.org/fileadmin/MULTIMEDIA/INSTITUTES/UIL/confintea/pdf/ GRALE/grale_sp.pdf

Ureña, G. y Valenzuela, J. (2011). Competencias Informáticas para el e-learning 2.0. RIED, 14(1), 137-160.

Vizoso, C. (2013). Los M.O.O.C.s un estilo de educación 3.0. En SCOPEO, MOOC: Estado de la situación actual, posibilidades, retos y futuro, 239-257. Salamanca:Universidad de Salamanca. http://scopeo.usal.es/wpcontent/uploads/2013/06/scopeoi002.pdf

Zapata-Ros, M. (2015). El diseño instruccional de los MOOC y el de los nuevos cursos abiertos personalizados. RED. Revista de Educación a Distancia, 45. http://www.um.es/ead/red/45/zapata.pdf 